\title{
Regulation of Interferon Regulatory Factor-3 by the Hepatitis C Virus Serine Protease
}

\author{
Eileen Foy, ${ }^{1}$ Kui Li, ${ }^{2}$ Chunfu Wang, ${ }^{1}$ Rhea Sumpter Jr., ${ }^{1}$ \\ Masanori Ikeda, ${ }^{2}$ Stanley M. Lemon, ${ }^{2}$ Michael Gale Jr. ${ }^{1 *}$
}

Persistent infections with hepatitis $\mathrm{C}$ virus ( $\mathrm{HCV}$ ) are likely to depend on viral inhibition of host defenses. We show that the HCV NS3/4A serine protease blocks the phosphorylation and effector action of interferon regulatory factor-3 (IRF-3), a key cellular antiviral signaling molecule. Disruption of NS3/4A protease function by mutation or a ketoamide peptidomimetic inhibitor relieved this blockade and restored IRF-3 phosphorylation after cellular challenge with an unrelated virus. Furthermore, dominant-negative or constitutively active IRF-3 mutants, respectively, enhanced or suppressed HCV RNA replication in hepatoma cells. Thus, the NS3/4A protease represents a dual therapeutic target, the inhibition of which may both block viral replication and restore IRF-3 control of HCV infection.

Persistent HCV infection is a leading cause of liver disease worldwide and is frequently refractory to current interferon (IFN)-based therapies $(1,2)$. HCV persistence is facilitated by the ability of the virus to incorporate adaptive mutations and to replicate as a population of genetically distinct quasispecies $(3,4)$, but is likely to result from specific disruption of host immune responses by $\mathrm{HCV}$ proteins (5-7). The HCV genome, a 9.6-kb single-stranded RNA of

A

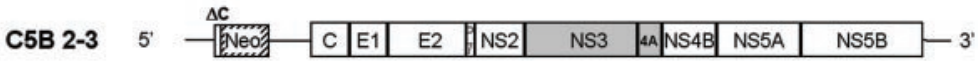

UHCV11
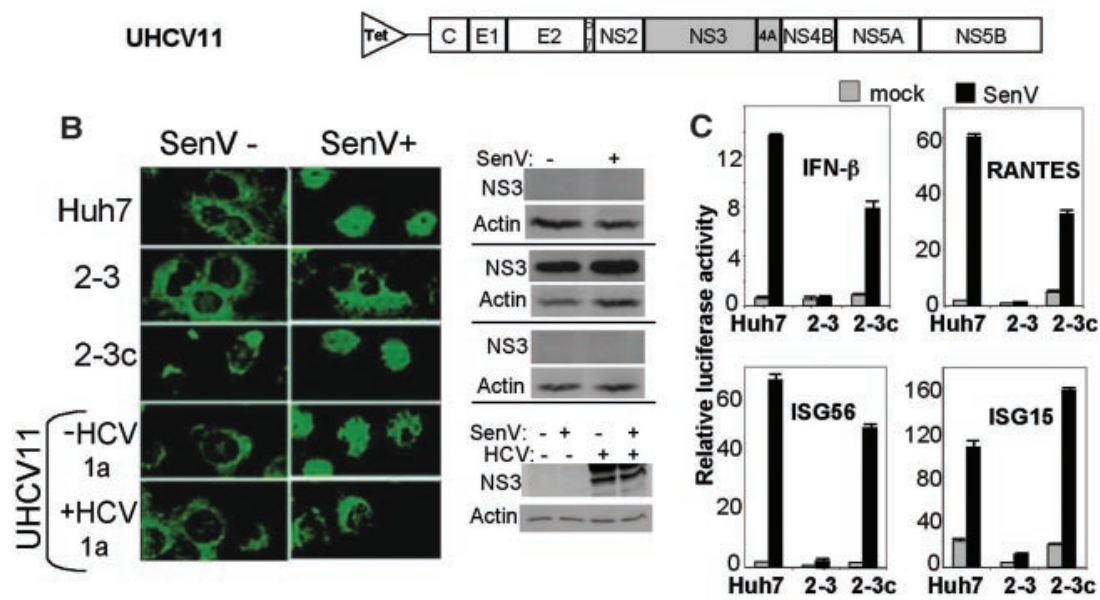

Fig. 1. HCV regulation of the IRF-3 pathway. (A) Organization of the genotype 1b HCV RNA replicating in Huh7 C5B2-3 cells (referred to as Huh7 2-3 cells) (19) and of the genotype 1a polyprotein expressed conditionally in UHCV11 cells. The NS3/4A coding region is shaded. (B) Immunostaining for IRF-3 in SenV-infected $\left(\mathrm{SenV}^{+}\right)$or mock-infected $\left(\mathrm{SenV}^{-}\right)$cells. From top to bottom, IRF-3 subcellular localization in control Huh7 cells, 2-3 cells, or interferon-cured 2-3c cells is shown (20), as are UHCV11 cells with (+HCV 1a) or without polyprotein expression (-HCV 1a). On the right are immunoblots for NS3 and actin in corresponding cell extracts. (C) Huh7 cells, Huh7 2-3 cells, or 2-3c cells were transfected with the indicated promoter-luciferase reporter constructs and then infected with SenV (black bars) or mock-infected (gray bars). Luciferase activities were determined in cell extracts (mean \pm S.D. from three experiments) (20). 
Fig. 2. The HCV NS3/4A protein complex blocks IRF-3 activation. (A) ISG56 promoter activities in Huh7 cells that were cotransfected with the ISG56 promoter-luciferase construct (Fig. 1C) and various HCV protein expression vectors, and then infected with SenV (black bars) or mock-infected (gray bars). The SenV-induced increase in luciferase activity within each culture was significantly different from cells expressing NS3/4A (Student's $t$ test; $P \leq 0.008$ ).
A

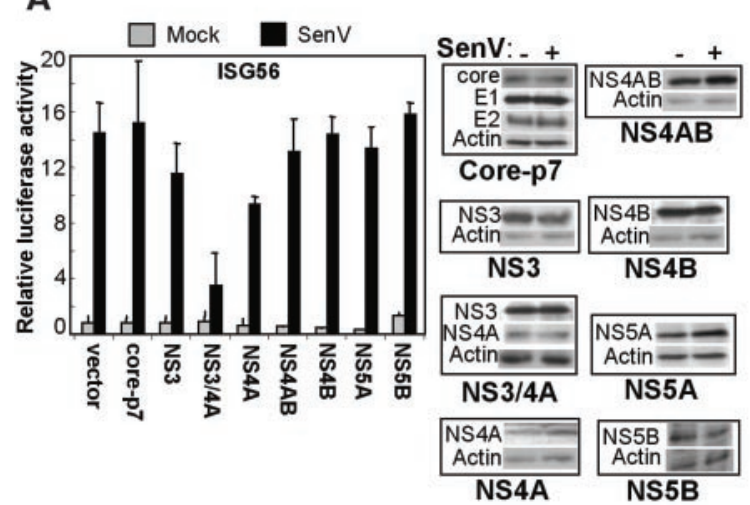

B

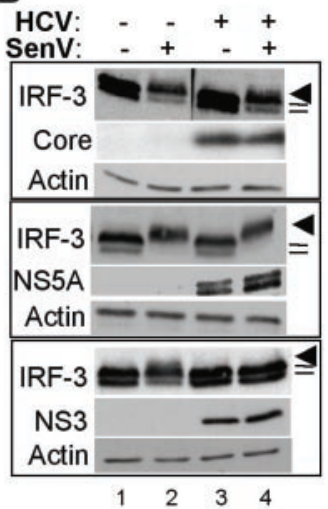

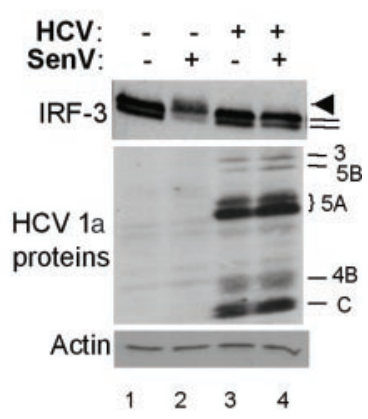

On the right are immunoblots of $\mathrm{HCV}$ proteins in the corresponding cell extracts. (B) Immunoblots (left) for IRF-3, HCV proteins, and actin in extracts of individual osteosarcoma cell lines cultured to repress $(-)$ or induce $(+)$ expression of the HCV core, NS5A, or NS3/4A proteins (denoted as HCV), and then either SenV-infected or mock-infected. The arrowhead and hatch marks denote high-mass hyperphosphorylated (virus-activated) and basally phosphorylated (inactive) IRF-3 isoforms, respectively. A similar analysis (right) of UHCV11 cells conditionally expressing the entire viral polyprotein. The core protein (labeled C) and NS3, NS5B, NS5A, and NS4B were detected by using a well-characterized patient serum $(17,20)$. (C) IRF-3 subcellular localization in osteosarcoma cells conditionally expressing core, NS5A, or NS3/4A in the presence or absence of SenV infection.

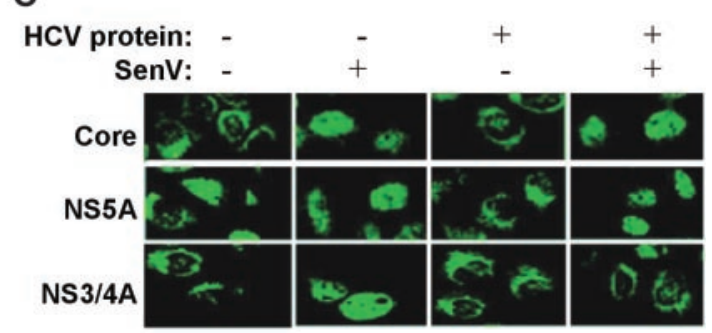

positive polarity, encodes a large polyprotein that is posttranslationally cleaved into structural and nonstructural (NS) proteins (Fig. 1A) (8). The NS proteins support viral RNA replication (9) and include the NS3/4A holoenzyme complex that has serine protease, adenosine triphosphatase (ATPase), and RNA helicase activities (10). The NS3/4A protease catalyzes the posttranslational processing of NS proteins from the viral polyprotein and is a prime target for the development of new antiviral drugs.

Cellular control of virus infection is mediated through a variety of processes that affect different stages of the viral life cycle (11). Interferon regulatory factors (IRFs) are key transcription factors that initiate this cellular antiviral state (12). IRF-3 is a latent cytoplasmic factor that is activated through phosphorylation by an undefined virus-activated kinase (VAK). VAK is stimulated by double-stranded RNA intermediates and other products of virus replication (13-15). Phosphorylated IRF-3 translocates to the nucleus, where it induces transcription of type I IFNs and other antiviral genes $(13,16)$. HCV RNA replication has the capacity to activate IRF-3 and to stimulate cellular antiviral responses (17), which suggests that control of IRF-3 could be an important determinant of viral persistence. Indeed, IRF3-null mice lack resistance to challenge with

'Department of Microbiology, University of Texas Southwestern Medical Center, Dallas, TX 753909048, USA. 'Department of Microbiology and Immunology, University of Texas Medical Branch, Galveston, TX 77555-1019, USA.

*To whom correspondence should be addressed. Email: Michael.Gale@UTSouthwestern.edu

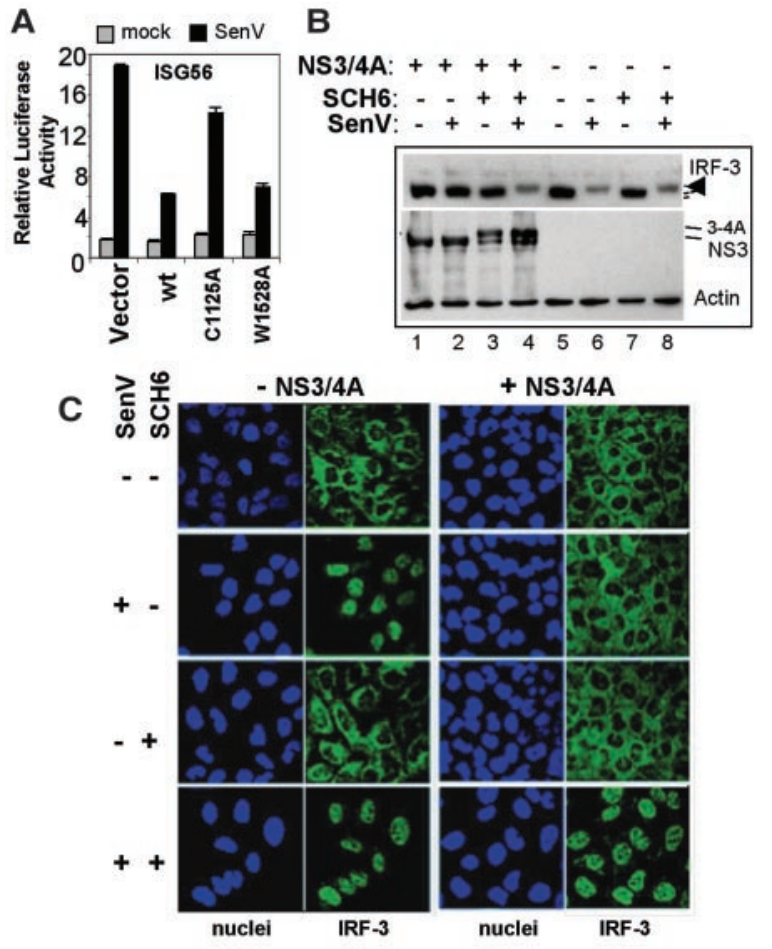

Fig. 3. The IRF-3 blockade is dependent on HCV NS3/4A protease activity and prevented by a small molecule inhibitor of viral protease function. (A) ISG56 promoter activity in Huh7 cells transiently expressing wt NS3/4A, or the C1125A or W1528A NS3/4A mutants. See Fig. 1C legend for details. (B) Immunoblots for IRF-3 (top gel) and NS3 (bottom gel) in osteosarcoma cells that were cultured to induce $(+)$ or repress $(-)$ $\mathrm{NS} 3 / 4 \mathrm{~A}$ expression in the presence or absence of $20 \mu \mathrm{M} \mathrm{SCH6}$, and then SenV-infected or mockinfected. (C) Subcellular localization of IRF-3 under the conditions described in Fig. 3B: left, DAPI staining of nuclei; right, FITC staining for IRF-3.

other viruses (18), and some viruses encode proteins perturbing IRF-3 function $(12,15)$.

Because HCV does not grow in cultured cells (8), we studied the IRF-3 pathway in Huh7 hepatoma cells containing an autonomously replicating, genome-length, genotype $1 \mathrm{~b} \mathrm{HCV}$ RNA (Huh7 2-3 cells) (19) and in osteosarcoma cells that conditionally express the entire genotype 1a polyprotein (UHCV11 cells) (Fig. 1A). Sendai virus $(\mathrm{SenV})$ is a potent inducer of
IRF-3 activation (13) When it infects control Huh7 cells, it induces nuclear translocation of IRF-3, thereby demonstrating the presence of an intact IRF-3 pathway in these cells (Fig. 1B). However, neither endogenous replicating $\mathrm{HCV}$ RNA nor SenV infection induced nuclear translocation of IRF-3 in Huh7 2-3 cells (Fig. 1B). Elimination of replicating HCV RNA by prior treatment with high concentrations of IFN- $\alpha 2 b$ (Huh7 2-3c cells) (20) restored SenV activation 

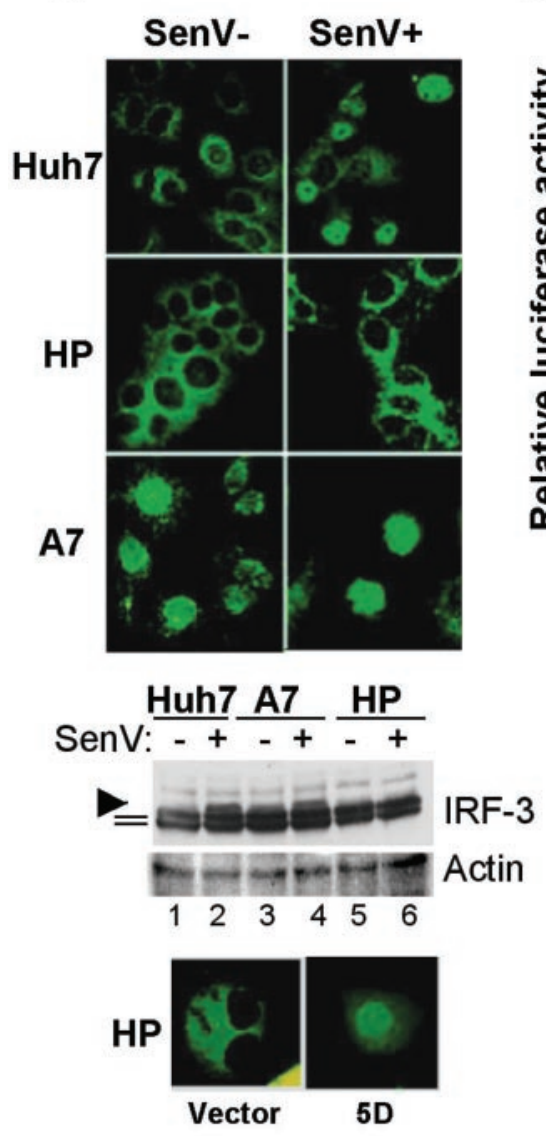

Fig. 4. The IRF-3 pathway regulates HCV RNA replication. IRF-3 localization, expression, and HCV RNA replication in normal Huh7 cells or Huh7 cells harboring HP or A7 subgenomic replicons. (A) IRF-3 localization (top) and protein expression (middle) within mock-infected or SenV-infected cells. (Bottom) IRF-3 localization within HP replicon cells after transient expression of

the IRF-3 5D mutant $(20,26)$. (B) IFN- $\beta$, ISG15, ISG56, or RANTES promoter activities in replicon cells infected with SenV. See legend of Fig. 1C for details. (C) NS5A, IRF-3, ISG56, and ISG15 protein expression in normal Huh7 cells (lane 1) or A7 (left gel) and HP replicon cells (right gel) after transfection with $1 \mu \mathrm{g}$ of vector control, or 0.5 or $1 \mu \mathrm{g}$ of IRF-3 5D or IRF-3 $\Delta \mathrm{N}$ expression plasmids, as indicated. Bands representing endogenous IRF-3, and 5D and $\Delta \mathrm{N}$ mutants are marked. Lane 3 in each gel shows protein levels in control cultures treated for 24 hours with $10 \mathrm{U} / \mathrm{ml}$ of IFN- $\alpha 2 \mathrm{a}$. (Bottom) Northern blots of HCV (upper) and glyceraldehyde 3-phosphate dehydrogenase (GAPDH) RNAs (lower) present in control Huh7 cells (lane 5), or A7 (lanes 6 to 10) and HP (lanes 11 to 15) replicon cells transfected with IRF-3 expression plasmids as indicated. Values below each lane indicate viral RNA abundance relative to the vector control.

of IRF-3 and showed that the inhibition of IRF-3 activation was HCV-specific. IRF-3 activation was also blocked by HCV polyprotein expression in UHCV11 cells (Fig. 1B), which indicates that IRF-3 function is blocked by multiple HCV genotypes and in different cell types, independently of HCV RNA replication. Neither HCV RNA replication nor conditional expression of $\mathrm{HCV}$ proteins inhibited $\mathrm{SenV}$ infection (21).

IRF-3 transcriptionally activates the promoters for the IFN- $\beta$, IFN-stimulated genes ISG56 and ISG15, and RANTES genes (22). We found each promoter to be induced efficiently in con- trol Huh7 cells upon SenV infection (Fig. 1C). In contrast, SenV induction of promoter activity was blocked in Huh7 2-3 cells containing replicating HCV RNA, but fully restored in interferon-cured Huh7 2-3c cells. Thus, disruption of the IRF-3 pathway by one or more HCV proteins blocks expression of IRF-3-activated genes. Although transient expression of the HCV core-E1-E2-p7, NS4B, NS4AB, NS5A or NS5B proteins did not affect SenV induction of the ISG56 promoter in Huh7 cells, expression of the NS3/4A complex strongly inhibited promoter induction (Fig. 2A), which suggested that it was responsible for perturbation of IRF-3 func- tion. Because the stability, subcellular localization, and function of NS3 depend on noncovalent association with NS4A (10), we assessed the inhibitory activity of NS3 and NS4A individually. Neither protein expressed alone substantially impaired induction of the ISG56 promoter (Fig. 2A). Further experiments in osteosarcoma cells demonstrated that NS3/4A disrupts IRF-3 function by blocking both accumulation of the hyperphosphorylated IRF-3 isoform (Fig. 2B) and the nuclear translocation of IRF-3 (Fig. 2C) resulting from viral induction of VAK (23).

To determine whether the enzymatic activities or RNA binding properties of NS3/4A are required for disruption of IRF-3 activation, we introduced single-amino acid substitutions into NS3 that eliminated the RNA binding and helicase functions [replacing $\operatorname{Trp}^{1528}$ with Ala (W1528A)] (24) or its serine protease activity [replacing Cys ${ }^{1125}$ with Ala (C1125A)] $(10,20)$. The NS3/4A complex containing the C1125A mutation lacked protease activity when expressed in Huh7 cells (21) and failed to block SenV induction of the IRF-3-dependent ISG56 promoter (Fig. 3A). In contrast, the W1528A mutant retained the ability to block IRF-3 activation (Fig. 3A). Because these results suggest that the serine protease activity of NS3 is responsible for perturbation of the IRF-3 pathway, we assessed the ability of SCH6, one of a class of recently described specific peptidomimetic ketoamide inhibitors of the NS3/4A serine protease (25), to reverse the NS3/4A inhibition of SenV-induced IRF-3 phosphorylation. The antiviral activity of SCH6 eliminated detectable HCV proteins and caused profound reductions in viral RNA abundance when the compound was added to cultures of Huh7 2-3 cells for 5 to 7 days (21). Consistent with its ability to inhibit the NS3/4A protease, SCH6 blocked cis-cleavage of the NS3/4A precursor protein in osteosarcoma cells (Fig. 3B). SCH6 also rescued SenV-induced IRF-3 phosphorylation and IRF-3 nuclear translocation in these cells (Fig. 3, $\mathrm{B}$ and $\mathrm{C}$ ). These data provide strong evidence that the NS3/4A serine protease activity is required for blockade of the IRF-3 pathway and that this blockade can be reversed by antiviral inhibitors of protease activity. We confirmed this directly by demonstrating the appearance of phosphorylated IRF-3 in Huh7 2-3 cells after treatment with SCH6 (21).

To further define the relationship between HCV replication and IRF-3, we characterized IRF-3 activation status and viral RNA replication in cells containing subgenomic HCV RNA replicons constructed from the wild-type Con1 HCV sequence (20). Wild-type Con1 RNA replicates poorly in Huh7 cells but accumulates adaptive mutations in the NS proteins that variably enhance its replication capacity $(9,19,26)$. One replicon, HP, exhibited robust replication and blocked both basal and SenV-induced IRF-3 phosphorylation, nuclear translocation, 
and IRF-3-dependent promoter activity (Fig. 4). Inhibition of nuclear translocation was not due to a global defect in transport, because a constitutively active, phospho-mimetic IRF-3 mutant, 5D (27), efficiently localized to the nucleus when expressed in these cells (Fig. 4A, bottom). A second Con1 replicon, A7, replicated poorly. A7 replicon cells had only $1 / 10$ th the viral RNA abundance of HP cells, and they displayed a low basal level of nuclear localized, hyperphosphorylated IRF-3 and IRF-3-dependent promoter activity that was further responsive to SenV challenge (Fig. 4, A and B). These results show that $\mathrm{HCV}$ replication has the potential to both activate and block the IRF-3 pathway.

The basal activation of IRF-3 in A7 cells correlated with expression of the IRF-3-dependent genes ISG56 and ISGI5 (Fig. 4C) (22). Transient expression of the constitutively active IRF-3 5D mutant (27) in these cells further increased ISGI5 and ISG56 expression and caused a modest reduction in replicon abundance. In contrast, expression of a dominant-negative IRF-3 mutant, $\Delta \mathrm{N}$ (27), resulted in a $>500 \%$ increase in HCV RNA levels and a parallel reduction in IRF-3 target gene expression. Thus, disruption of the IRF-3 pathway enhances HCV RNA replication, which may explain the higher basal abundance of HCV RNA in HP than in A7 cells (Fig. 4C, bottom, compare lanes 6 and 11). Although expression of the constitutively active 5D mutant induced IRF-3 target gene expression and reduced replicon abundance in HP cells, expression of the dominant-negative $\Delta \mathrm{N}$ mutant had no effect in these cells because of the preexisting block in the IRF-3 pathway imposed by NS3/4A.

Our results show that $\mathrm{HCV}$ interacts directly with host pathway(s) that signal IRF-3 activation and that HCV, like other viruses (28), has evolved a specific mechanism to circumvent a major arm of the host's immune response. Activated IRF-3 suppresses HCV RNA replication, whereas viral RNA abundance is increased when IRF-3 function is disrupted. These findings make the activation status of IRF-3 an important factor in the virus-host cell interaction. The inhibition of IRF-3 activation by the NS3/4A protease likely involves perturbation of cellular processes that catalyze or otherwise trigger IRF-3 phosphorylation (27). Although the VAK responsible for phosphorylation of IRF-3 in this context has yet to be identified, our results suggest that one or more signaling components in the IRF-3 activation pathway, including possibly the VAK itself, could be specifically targeted for proteolysis by NS3/4A.

The regulatory factor IRF-3 induces expression of a variety of cellular genes including the type I IFNs $(14,29)$, which contribute to and further amplify the antiviral response by inducing hundreds of IFN-stimulated genes $(11,28)$. Inhibition of IRF-3 activation may not only promote viral persistence after initial infection, but also may reduce the effectiveness of interferon therapies, because many IFN-stimulated genes contain IRF-3 target sites within their promoter/enhancer regions $(22,29)$. Our results with SCH6 suggest that candidate antivirals that target the HCV protease, while blocking viral replication by interfering with processing of the polyprotein, may also restore the responsiveness of the IRF-3 pathway. Such "dual efficacy" should be considered in evaluating protease inhibitors destined for future clinical trials.

\section{References and Notes}

1. M. W. Fried et al., N. Engl. J. Med. 347, 975 (2002).

2. J. G. McHutchison, K. Patel, Hepatology 36, S245 (2002).

3. X. Forns, J. Bukh, Clin. Liver Dis. 3, 693 (1999).

4. P. Farci et al., Proc. Natl. Acad. Sci. U.S.A 99, 3081 (2002).

5. D. R. Taylor, S. T. Shi, P. R. Romano, G. N. Barber, M. M. C. Lai, Science 285, 107 (1999).

6. J. Pflugheber et al., Proc. Natl. Acad. Sci. U.S.A. 99 4650 (2002).

7. N. Zhu, C. F. Ware, M. M. Lai, Virology 283178 (2001).

8. K. Reed, C. Rice, in Hepatitis C Virus, H. Reesink, Ed. (Karger, Basel, 1998), pp. 1-39.

9. V. Lohmann et al., Science 285, 110 (1999).

10. R. De Francesco, C. Steinkuhler, Curr. Top. Microbiol. Immunol. 242, 149 (2000).

11. M. G. Katze, Y. He, M. Gale Jr., Nature Rev. Immunol. 2, 675 (2002)

12. B. Barnes, B. Lubyova, P. M. Pitha, J. Interferon Cytokine Res. 22, 59 (2002).

13. R. Lin, C. Heylbroeck, P. M. Pitha, J. Hiscott, Mol. Cell. Biol. 18, 2986 (1998).

14. M. Yoneyama et al., EMBO J. 17, 1087 (1998).
15. E. J. Smith, I. Marie, A. Prakash, A. Garcia-Sastre, D. E. Levy, J. Biol. Chem. 276, 8951 (2001).

16. N. C. Reich, J. Interferon Cytokine Res. 22, 103 (2002).

17. B. Fredericksen et al., Viral Immunol. 15, 29 (2001).

18. M. Sato et al., Immunity 13, 539 (2000).

19. M. Ikeda, M. Yi, K. Li, S. M. Lemon, J. Virol. 76, 2997 (2002).

20. Materials and methods are available as supporting material on Science Online.

21. S. M. Lemon, M. Gale Jr., unpublished results.

22. N. Grandvaux et al., J. Virol. 76, 5532 (2002).

23. M. J. Servant et al., J. Biol. Chem. 355 (2001).

24. C.-L. Tai et al., J. Virol. 75, 8289 (2001).

25. J. Edwin et al., International Patent Application WO02/08244 (2002).

26. K. J. Blight, A. A. Kolykhalov, C. M. Rice, Science 290, 1972 (2000)

27. R. Lin, Y. Mamane, J. Hiscott, Mol. Cell Biol. 19, 2465 (1999).

28. G. C. Sen, Annu. Rev. Microbiol. 55, 255 (2001).

29. T. Nakaya et al., Biochem. Biophys. Res. Commun. 283, 1150 (2001).

30. Supported by NIH grants U01-AI48235 (M.G.) and U19-Al40035 (S.M.L.), the Ellison Medical Foundation (ID-NS-0032), a gift from Mr. and Mrs. R. Batcheldor (M.G.), and a research grant from Schering-Plough (S.M.L.). M.G. is the Nancy C. and Jeffrey A. Marcus Scholar in Medical Research in Honor of Dr. Bill S. Vowell. K.L. was supported by the J. W. McLaughlin Fellowship. We thank S. Bogen, M. Murray, and B. Malcolm of the Schering-Plough Research Institute for kindly providing $\mathrm{SCH} 6$, and B. Fredericksen, W. Bresnahan, G. Klimpel, and L. Soong for critical review of the manuscript.

\section{Supporting Online Material}

www.sciencemag.org/cgi/content/full/1082604/DC1 Materials and Methods

References

21 January 2003; accepted 24 March 2003

Published online 17 April 2003;

10.1126/science. 1082604

Include this information when citing this paper.

\title{
Triggering the Interferon Antiviral Response Through an IKK-Related Pathway
}

\section{Sonia Sharma,* Benjamin R. tenOever,* Nathalie Grandvaux, Guo-Ping Zhou, Rongtuan Lin, $\uparrow$ John Hiscott $\dagger$}

\begin{abstract}
Rapid induction of type I interferon expression, a central event in establishing the innate antiviral response, requires cooperative activation of numerous transcription factors. Although signaling pathways that activate the transcription factors nuclear factor $\mathrm{KB}$ and ATF-2/c-Jun have been well characterized, activation of the interferon regulatory factors IRF-3 and IRF-7 has remained a critical missing link in understanding interferon signaling. We report here that the IKB kinase (IKK)-related kinases IKKE and TANK-binding kinase 1 are components of the virus-activated kinase that phosphorylate IRF-3 and IRF-7. These studies illustrate an essential role for an IKK-related kinase pathway in triggering the host antiviral response to viral infection.
\end{abstract}

The success of host cell defense against viral infection depends on detection of the invading pathogen. Upon recognizing viral antigens, the cell activates a multitude of signaling cascades to produce cytokines that both impede pathogen replication and stimulate immune responses $(1-3)$. Interferons (IFNs) are well-characterized components of the innate host defense, and rapid induction of IFN expression in response to viral infection requires posttranslational modification of transcription factors, including nuclear factor $\mathrm{kB}$ (NF-KB), ATF-2/c-Jun, and interferon regulatory factors (IRFs) IRF-3 and IRF-7 $(4,5)$. 


\section{Science}

\section{Regulation of Interferon Regulatory Factor-3 by the Hepatitis C Virus Serine Protease}

Eileen Foy, Kui Li, Chunfu Wang, Rhea Sumpter Jr., Masanori Ikeda, Stanley M. Lemon and Michael Gale Jr.

Science 300 (5622), 1145-1148.

DOI: 10.1126/science.1082604originally published online April 17, 2003

ARTICLE TOOLS

SUPPLEMENTARY MATERIALS

RELATED

CONTENT

REFERENCES

PERMISSIONS http://science.sciencemag.org/content/300/5622/1145

http://science.sciencemag.org/content/suppl/2003/05/15/1082604.DC1

http://stke.sciencemag.org/content/sigtrans/2003/183/tw198.abstract

This article cites 24 articles, 12 of which you can access for free http://science.sciencemag.org/content/300/5622/1145\#BIBL

http://www.sciencemag.org/help/reprints-and-permissions 